\title{
Fatal dapsone agranulocytosis in a Melanesian
}

\author{
P BARSS \\ Provincial Hospital, Alotau, Milne Bay Province, Papua New \\ Guinea*
}

Accepted for publication 10 July 1985

\begin{abstract}
Summary The death from agranulocytosis and septicaemia of a young Melanesian male receiving unsupervised treatment with $100 \mathrm{mg}$ daily of dapsone for indeterminate leprosy is reported. The historical recognition and clinical management of agranulocytosis resulting from dapsone is discussed. Careful observation of leprosy patients for side-effects during the initial weeks of treatment is recommended. This may include hospital admission and regular blood counts when possible.
\end{abstract}

\section{Introduction}

Sulphones were first noted to be effective for leprosy in rats in $1941 .{ }^{1}$ Clinical trials in humans followed soon after; however, it was not until 1958 that it was clearly recognized that agranulocytosis can result from the use of dapsone. ${ }^{2}$ A patient was described who recovered from agranulocytosis which had occurred after the administration of $125 \mathrm{mg}$ of dapsone daily for dermatitis herpetiformis. In 1970, a more alarming report was published ${ }^{3}$ concerning 16 US soldiers in Vietnam who developed agranulocytosis after receiving $25 \mathrm{mg}$ of dapsone daily as malaria prophylaxis for periods of from 3 weeks to 3 months; 8 died. At least 4 of the fatal cases had septicaemia with positive blood cultures for pseudomonas. Two had received kanamycin before their death. A further case of agranulocytosis was reported in an Australian soldier in Vietnam in 1970; he had also been taking 25 $\mathrm{mg}$ of dapsone daily for 4 months. This patient developed an infection in a bayonet wound caused by pseudomonas and also had pseudomonas septicaemia. He recovered following treatment with colistin. At the time these cases occurred, dapsone was being given routinely as an anti-malarial to many thousands of servicemen in Vietnam. This practice was stopped when the risks of agranulocy-

* Present address: School of Hygiene and Public Health, Johns Hopkins University, Baltimore, MD 21205, USA 
tosis from dapsone became apparent. However, more recently agranulocytosis has been reported as a complication of weekly Malaprim (100 mg dapsone, 12.5 pyrimethamine) prophylaxis. ${ }^{5}$

This paper reports the death of a young Melanesian male from agranulocytotosis. A clinical diagnosis of leprosy had been made at a heath centre. The patient was then immediately discharged home and took a course of dapsone without supervision.

\section{Case history}

A 23-year-old man was seen at a rural health centre with skin lesions. The diagnosis of leprosy was considered. A skin smear was negative. A biopsy was later reported to be suggestive of indeterminate leprosy. He was started on dapsone, $100 \mathrm{mg}$ daily. Because he wanted to go off and build a boat, the health centre staff released him to take treatment on his own. During the treatment he noted headache, body aches, jaundice, fever and dark urine. He presented himself to Alotau Hospital 48 days after starting treatment.

\section{FINDINGS}

On admission, he had a temperature of $38^{4}$, a pulse of 26 , and his blood pressure was $100 / 60$. A few crepitations were noted in the left chest, together with hepatosplenomegaly and tender left inguinal lymph nodes. Possible diagnoses considered by the admitting doctors were leprosy reaction, malaria, hepatitis and bacteraemia. The patient was given procaine penicillin and chloroquine. Laboratory tests were ordered. The patient deteriorated rapidly and at $17 \mathrm{~h}$ after admission the pulse was 100 and respirations 44 . He was then given intravenous fluids, crystalline penicillin, and chloramphenicol. He began complaining of chest pain, and coughed up green liquid sputum, became increasingly tachypnoeic and hypotensive, and had green liquid diarrhoea. He died $26 \mathrm{~h}$ after admission.

Results of blood tests taken before death were reviewed the following day. Haemoglobin was $11 \mathrm{~g} / 100 \mathrm{ml}$, white cell count $700 / \mathrm{mm} .{ }^{3}$ The blood smear showed many fragmented red cells and schistocytes, with very few neutrophils. The bilirubin was $1.8 \mathrm{mg} \%$. A wet stool preparation showed pus cells and erythrocytes, but no amoebae. A blood smear for malaria was negative. Due to the uncertain cause of sudden death, the coroner was notified, and a post-mortem was ordered. Bilateral pneumonia with consolidation was found.

A microscopic examination of the liver showed only oedema and increased bilirubin pigment. There was no hepatitis. The spleen was reported as normal. Cultures taken from the lung at autopsy grew Pseudomonas aeruginosa, resistant to all antibiotics except gentamicin and carbenacillin. 


\section{Discussion}

This patient died of a pseudomonas pneumonia which arose in the presence of severe agranulocytosis; septicaemia was also likely. The agranulocytosis was presumably a toxic effect of the dapsone therapy, which had been unsupervised. It is unknown whether the patient was taking the prescribed dosage of dapsone. It is possible that he was taking a greater dose than recommended, hoping to improve more rapidly. Although he felt unwell during the treatment, he did not report to the health staff until the day prior to his death.

Although agranulocytosis resulting from dapsone treatment is probably relatively rare, it would seem wise to keep new leprosy patients under close observation after initiating treatment, since other reactions to dapsone, as well as leprosy reactions, commonly occur in the first weeks to months of taking the drug. Because treatment of leprosy is sometimes toxic and can be lengthy, I also believe it is wise to take a skin biopsy when new patients are first seen to confirm the diagnosis and type of leprosy. This information will be useful to sceptical health workers later in treatment, when obvious signs of the type of leprosy involved may no longer be visible. Other side-effects attributed to dapsone commonly include haemolytic anaemia, and only rarely methaemoglobinaemia, various skin disorders, hepatotoxicity, acute psychosis, and headache. I therefore monitor haemoglobin or haematocrit levels and white cell counts weekly in leprosy patients during the first couple of months of therapy.

Early symptoms of agranulocytosis include abrupt onset of sore throat, fever, chills, malaise, fatigue and weakness. Inflammation of the throat is said to soon progress to necrosis, with formation of a membrane and subsequent ulceration. Diagnostic criteria for drug-induced agranulocytosis include a neutrophil concentration of less than $0 \cdot 2 \times 10^{y} / 1$, and recovery in the 14 days after withdrawal of the drug. ${ }^{6}$ If a patient does develop agranulocytosis, death, if it occurs, is due to infection, particularly with pneumonia and/or septicaemia. In managing a patient with this disorder, cultures of blood, sputum and any wounds should be taken if possible. In view of the common occurrence of pseudomonas infections, prophylactic administration of an aminoglycoside antibiotic, such as gentamicin, is advisable. Other organisms which cause sepsis in such patients include E. coli, Proteus and Staphylococcus aureus. Initial treatment with a broad spectrum penicillin or cephalosporin, probenecid and perhaps metronidazole is also recommended until results of cultures are known. Isolation of the patient is important, ${ }^{6}$ and treatment of shock with fluids, and good oral hygiene are also advised. ${ }^{7}$

Agranulocytosis has been reported to have a mortality of about $20 \%$ or more. De Gruchy commented that this is a disturbingly high mortality, when one considers that agranulocytosis is self-limiting following withdrawal of the causative drug. Leukaemoid reactions are seen occasionally during the recovery phase. $^{8}$ 
Finally, although chronic mycobacterial diseases such as leprosy and tuberculosis can often be managed successfully by non-specialists in rural hospitals and health centres, various complications do arise relatively frequently during the initial phase of treatment. It is therefore probably wise to admit such patients to hospital or a health centre during the first few weeks of treatment, if space is available. This is particularly important if the patient is unreliable or comes from a remote village. Regular observation is essential. If patients deteriorate during treatment, temporary cessation of all therapy is advisable, until drug toxicity and other reactions have been carefully excluded. Such careful and cautious management could, I believe, prevent many of the numerous deaths and complications which do occur during treatment of leprosy and tuberculosis.

\section{References}

1 Weinstein L. Drugs used in the chemotherapy of tuberculosis and leprosy. In The Pharmacological Basis of Therapeutics. Goodman LS, Gilman A (eds), Macmillan. New York 1975; p. 1216.

${ }^{2}$ McKenna WB, Chalmers AC. Agranulocytosis following dapsone therapy. Br Med J, 1958; 1: 324-5.

${ }^{3}$ Ognibene AJ. Agranulocytosis due to dapsone. Ann Int Med, 1970; 72: 521-4.

${ }^{4}$ Stickland JF. Agranulocytosis probably due to dapsone in an infantry soldier. Med J Aust, 1970; May 9: 959-60.

5 Nicholls MD, Concannon AJ. Malaprim-induced agranulocytosis and red-cell aplasia. Med J Aust, 1982; 2: 564-6.

6 Young GAR, Vincent PC. Drug-induced agranulocytosis. Clinics in Haematology, 1980; 9: 483504.

7 De Gruchy GC. Drug-Induced Blood Disorders. Blackwell. Oxford 1975; 76-117.

${ }^{8}$ Levine $\mathrm{PH}$, Weintraub LR. Pseudoleukemia following recovery from dapsone-induced agranulocytosis. Ann Intern Med, 1968; 68: 1060. 